\title{
Assessment of neovascularization of carotid artery atherosclerotic plaques using superb microvascular imaging: a comparison with contrast-enhanced ultrasound imaging and histology
}

\author{
Qi Meng" ${ }^{1 \#}$ Xia Xie ${ }^{1 \#}, \mathrm{Li} \mathrm{Li}^{2}$, Chao Jiang ${ }^{3}$, Keqiang Zhao ${ }^{3}$, Zhiyong Bai ${ }^{1}$, Zhuozhao Zheng ${ }^{4}$, Yu Yang ${ }^{3}$, \\ Yan Yu ${ }^{2}$, Huabin Zhang $^{1}$, Xihai Zhao \\ ${ }^{1}$ Department of Ultrasound, Beijing Tsinghua Changgung Hospital, School of Clinical Medicine, Tsinghua University, Beijing, China; ${ }^{2}$ Department \\ of Pathology, Beijing Tsinghua Changgung Hospital, School of Clinical Medicine, Tsinghua University, Beijing, China; ${ }^{3}$ Department of Vascular \\ Surgery, Beijing Tsinghua Changgung Hospital, School of Clinical Medicine, Tsinghua University, Beijing, China; ${ }^{4}$ Department of Radiology, \\ Beijing Tsinghua Changgung Hospital, School of Clinical Medicine, Tsinghua University, Beijing, China; ${ }^{5}$ Center for Biomedical Imaging Research, \\ Department of Biomedical Engineering, Tsinghua University School of Medicine, Beijing, China \\ \#These authors contributed equally to this work.
}

Correspondence to: Huabin Zhang, MD. Department of Ultrasound, Beijing Tsinghua Changgung Hospital, School of Clinical Medicine, Tsinghua University, No. 168 Litang Road, Changping District, Beijing 102218, China. Email: huabinzhang@bjmu.edu.cn; Xihai Zhao, MD, PhD. Center for Biomedical Imaging Research, Tsinghua University School of Medicine, Haidian District, Beijing 100084, China. Email: xihaizhao@tsinghua.edu.cn.

Background: This study aimed to investigate the usefulness of superb microvascular imaging (SMI), a novel non-contrast-enhanced ultrasound technique, in characterizing neovessels within carotid atherosclerotic plaques through comparison with contrast-enhanced ultrasound (CEUS) and histology.

Methods: Patients with carotid plaque were recruited and underwent SMI and CEUS ultrasound imaging of the carotid arteries. The maximum plaque thickness, length, and stenosis of each plaque were measured. Grade of the neovessels was determined by SMI and CEUS, respectively. Grade 0 was defined as no blood flow signal/microbubbles within plaques; grade 1 was defined as moderate blood flow signals/ microbubbles confined to the shoulder and/or adventitial side of the plaque; and grade 2 was defined as extensive intraplaque signals/microbubbles. Patients with symptomatic carotid stenosis (stenosis $\geq 50 \%$ ) or asymptomatic carotid stenosis (stenosis $\geq 70 \%$ ) underwent endarterectomy, and plaque specimens were subjected to immunohistochemical analysis of CD31 expression. The neovessels were quantified by histology. The agreement of SMI with CEUS and histology in characterizing neovessels was analyzed using weighted Kappa statistic and Spearman's correlation analyses.

Results: Seventy-eight patients (mean age: $67.3 \pm 8.9$ years old, 63 males) were recruited. Of these patients, $52(66.7 \%)$ had a unilateral plaque and $26(33.3 \%)$ had bilateral plaques in the carotid arteries. For the 104 carotid plaques detected, the mean plaque thickness and length were $4.3 \pm 1.1$ and $18.8 \pm 6.6 \mathrm{~mm}$, respectively. The prevalence of $<50 \%, 50-69 \%$, and $\geq 70 \%$ stenosis was $43.3 \%, 24.0 \%$, and $32.7 \%$, respectively. Excellent agreement was found between SMI and CEUS ( $\kappa=0.825$ at the plaque level; $\kappa=0.820$ at the patient level) in evaluating the neovessel grade within the carotid plaques. Of the 25 patients who underwent carotid endarterectomy, a strong correlation $(r=0.660, \mathrm{P}<0.001)$ was found between SMI and histology in the evaluation of intraplaque neovessels. SMI had excellent scan-rescan $(\kappa=0.857)$, intra-reader $(\kappa=0.810)$, and inter-reader $(\kappa=0.754)$ agreement in the assessment of intraplaque neovessels.

Conclusions: The SMI technique is capable of reliably characterizing neovessels within carotid atherosclerotic plaques and demonstrates good to excellent agreement with histology and CEUS.

Keywords: Carotid arteries; atherosclerosis; neovascularization; ultrasonography 
Submitted Aug 01, 2020. Accepted for publication Nov 20, 2020.

doi: 10.21037 /qims-20-933

View this article at: http://dx.doi.org/10.21037/qims-20-933

\section{Introduction}

Carotid atherosclerotic vulnerable plaque has been demonstrated to be one of the major causes of ischemic cerebrovascular events (1). Neovascularization within the atherosclerotic plaque is well evidenced to play an important role in plaque vulnerability and acts as a major source of intraplaque hemorrhage (2). Thus, accurate characterization of neovascularization within carotid plaques is vital. Although Doppler ultrasound and computed tomography angiography are the most commonly used modalities for the assessment of carotid atherosclerosis, these approaches cannot depict the neovasculature within carotid plaques (3-5). In contrast, contrast-enhanced ultrasound (CEUS) and dynamic contrast-enhanced magnetic resonance imaging (DCE-MRI) are capable of characterizing the neovascularization of carotid plaques (6-11). However, CEUS examination depends on the use of phospholipidstabilized sulfur hexafluoride microbubbles, which are contraindicated in patients with severe chronic obstructive pulmonary disease or asthma. DCE-MRI depends on the use of a gadolinium-based contrast agent that can only be administered to patients without renal dysfunction and contrast allergies. Also, due to the necessity of repeated administration of a contrast agent, CEUS and DCE-MRI are not the ideal modalities for monitoring the changes of neovessels within plaques during treatment $(12,13)$.

In 2014, a non-contrast-enhanced ultrasound technique of superb microvascular imaging (SMI; Toshiba Medical Systems Corporation, Tochigi, Japan), was proposed for the in vivo assessment of microvessels. The imaging mechanism of SMI is based on the movement of blood cells in blood vessels rather than the actual blood vessel diameter and is assessed by high sensitivity Doppler detection and special filtering technology. SMI displays blood vessels having a much larger diameter than they actually have which is an overflow effect. SMI utilizes an adaptive algorithm to distinguish true low-velocity flow from clutter. As a result, without the use of contrast agents, SMI is able to visualize minute vessels with low-velocity flow signals, which are usually omitted by conventional Doppler imaging. There are two imaging modes in SMI: monochrome
(mSMI) and color (cSMI) modes (14). In previous studies evaluating microvessels in thyroid nodules (15-17), breast lesions (18-21), small hepatocellular carcinomas (22), and neonatal brain parenchyma (23), SMI depicted greater details of the microvascular flow and vessel branches than conventional non-contrast ultrasound techniques (color and power Doppler imaging). SMI may have the potential to characterize neovessels within carotid atherosclerotic plaques (24). Most recently, investigators demonstrated that there was fair to good agreement between SMI and CEUS and excellent agreement between SMI and histology in the evaluation of neovessels within carotid plaques (25-27). Nevertheless, there is a lack of validation for the usefulness of SMI compared with both CEUS and histology in assessing the neovessels of carotid plaques.

This study sought to investigate the usefulness of SMI in characterizing neovessels within carotid atherosclerotic plaques through comparison with CEUS and histology.

\section{Methods}

\section{Study sample}

From January 2015 to May 2018, we retrospectively reviewed the carotid ultrasound database at Beijing Tsinghua Changgung Hospital. Patients with large atherosclerotic plaque in their carotid arteries (maximum plaque thickness $>2.5 \mathrm{~mm}$ ) determined by grayscale ultrasound imaging were recruited in this study. Subjects with the following conditions were excluded: (I) severe multiple organ failure; (II) hypersensitivity to albumin, blood-derived products, or the ultrasound imaging contrast agent (SonoVue); and (III) carotid plaques with acoustic shadowing caused by heavy calcification, which can influence the analysis of microbubbles in CEUS examinations. All included subjects underwent SMI and CEUS examinations. Clinical data including age, sex, body mass index (BMI), history of hypertension, diabetes mellitus, smoking, hyperlipidemia, stroke, coronary heart disease (CHD), cholesterol levels, and blood pressure were collected from patients' medical records. The study protocol was approved by the ethics committee of Beijing Tsinghua Changgung Hospital and 
informed consent was obtained from all participants.

\section{Ultrasound imaging}

Ultrasound imaging was performed for carotid arteries with large atherosclerotic plaques by a sonographer with more than 5 years of experience in vascular ultrasound imaging. Pre- and post-contrast imaging was conducted on an ultrasound scanner (Aplio500, Toshiba, Japan) using a 3-9 MHz transducer. All plaques were scanned dynamically in grayscale mode in longitudinal and transverse sections in order to measure maximum plaque thickness and length of each plaque. According to the Society of Radiologists in Ultrasound Consensus Conference, luminal stenosis was measured using grayscale and Doppler ultrasound (28). The SMI images of each plaque were acquired using the SMI acquisition mode with close attention paid to the previously identified plaques at multiple different sections and orientations. Grayscale and mSMI were visualized simultaneously using the double-screen function. SMI was performed with a mechanical index of 1.5 , a frame rate of $25-30 \mathrm{fps}$, a velocity range of $1.0-1.5 \mathrm{~cm} / \mathrm{s}$, and a dynamic range of 55-60 dB. The SMI examination was accomplished within 1 minute for each plaque, and a video of the target plaque was recorded. Carotid CEUS was carried out by injecting a $2 \mathrm{~mL}$ bolus of microbubble agent, SonoVue (Bracco Imaging, Milan, Italy) via the median cubital vein, followed by a $5 \mathrm{~mL}$ normal saline flush. The plaque region was imaged for 100 seconds in total -10 seconds before and 90 seconds after the administration of the SonoVue microbubbles. During the examination period, the transducer was held still at the center of the plaques. Other CEUS settings included a mechanical index of 0.1 , a frame rate of 10-15 fps, and a dynamic range of 50-60 dB. Both static images and dynamic real-time videos of SMI and CEUS were stored for further off-line analysis.

\section{Image analysis}

Plaques were classified by a radiologist who had more than 5 years' experience in vascular ultrasound imaging. Plaques were divided into five types according to echogenicity on the grayscale ultrasound images using the following criteria: type I: uniformly anechoic plaque; type II: predominantly hypoechoic plaque or anechoic areas $>50 \%$; type III: predominantly echogenic plaque with $<50 \%$ hypoechoic areas; type IV: uniformly echogenic plaque; and type V: unclassified plaques due to acoustic shadowing caused by heavy calcification (29,30). The acquired CEUS and SMI data were evaluated independently by two radiologists who had more than 5 years of experience in vascular ultrasound imaging blinded to the histology results. The degree of contrast enhancement of carotid plaques was graded as: grade 0: no visible microbubbles within the plaque; grade 1: moderate microbubbles confined to the shoulder and/ or adventitial side of the plaque; and grade 2: extensive intraplaque enhancement (31). The section with the maximal number of neovessels from the repeated SMI scans at multiple orientations was chosen for the classification of the intraplaque neovessels. The grading criteria for blood flow signals by SMI are: grade 0: no visible blood flow signal within the plaque; grade 1: moderate blood flow signals confined to the shoulder and/or adventitial region of the plaque; and grade 2: extensive intraplaque blood flow signals.

\section{Reproducibility study}

Fifteen plaques were randomly selected to determine the scan-rescan, intra-observer, and inter-observer reproducibility in evaluating the grade of blood flow signals within plaques using SMI. To minimize the memory bias, a 2-month time interval was set to test the intra-observer reproducibility.

\section{Histology}

Subjects who had symptomatic carotid stenosis (stenosis $\geq 50 \%$ ) or asymptomatic carotid stenosis (stenosis $\geq 70 \%$ ) underwent endarterectomy surgery. Specimens were collected, processed, and analyzed by histology. The excised plaques were fixed in $10 \%$ buffered formalin, decalcified, and embedded in paraffin. Transverse $4-\mu \mathrm{m}$ sections were obtained at $2.0-\mathrm{mm}$ intervals. The excised plaques were cut from the proximal to distal portions, and all histological slices were retained except severely damaged ones. Immunohistochemical analysis was performed with CD31, a monoclonal antibody for endothelial cells, by a pathologist who was blinded to the clinical characteristics and the results of the ultrasound examination. The neovessels were then counted under a high-power microscope (200x). The mean neovessel density on histology was quantified as the 
number of neovessels $/ \mathrm{mm}^{2}$ and was used for determining the correlation with the imaging data.

\section{Statistical analysis}

The continuous variables were presented as mean \pm standard deviation (SD) and the discrete variables were described as percentages. The agreement between SMI and CEUS and between SMI and histology in assessing neovessels was analyzed using weighted Kappa and Spearman's correlation tests, respectively. For those patients without histology specimens, we utilized CEUS as the reference standard for assessing intraplaque neovessels. The agreement was rated using the following criteria: poor, weighted $\kappa<0.4$; fair, weighted $\kappa=0.4-0.6$; good, weighted $\kappa=0.6-0.75$; and excellent, weighted $\kappa>0.75$. The reproducibility of SMI in evaluating neovessels was analyzed with Cohen's Kappa and weighted Kappa tests. A P value $<0.05$ was considered statistically significant. All statistical analyses were performed using SPSS version 24.0 (IBM, Chicago, IL, USA).

\section{Results}

A total of 92 patients were included in this study between January 2015 and May 2018. Of them, 14 patients were excluded due to acoustic shadowing in the carotid plaques caused by heavy calcification (type $\mathrm{V}$ plaques). Of the remaining 78 patients, 59 (75.6\%) had hypertension, 46 (59.0\%) had hyperlipidemia, $39(50.0 \%)$ had a history of smoking, 36 (46.2\%) had CHD, 28 (35.9\%) had diabetes mellitus, and $23(29.5 \%)$ had a history of stroke. The patient recruitment process is detailed in Figure 1. Patient clinical characteristics are listed in Table 1.

\section{Characteristics of carotid plaques on ultrasound imaging}

Of the total sample of 78 patients, $52(66.7 \%)$ had a solitary plaque in a unilateral carotid artery, and $26(33.3 \%)$ had two plaques in bilateral carotid arteries. The 104 carotid plaques detected overall had a mean plaque thickness and length of $4.3 \pm 1.1$ and $18.8 \pm 6.6 \mathrm{~mm}$, respectively. The prevalence of $<50 \%, 50-69 \%$, and $\geq 70 \%$ stenosis was $43.3 \%, 24.0 \%$, and $32.7 \%$, respectively. According to the echo, the 104 plaques were classified into the following types: type I, 40 (38.5\%); type II, 43 (41.3\%); type III, 13 (12.5\%); and type IV, 8
(7.7\%). Type $\mathrm{V}$ plaques were not included in the analysis due to the omission of plaques with acoustic shadowing caused by calcification. The characteristics of the plaques are documented in Table 2 .

\section{Agreement between SMI and CEUS}

The results of the agreement between SMI and CEUS in determining the grade of neovessels are shown in Table 3. Weighted kappa analysis revealed excellent agreement ( $\kappa=0.825,95 \%$ CI: 0.739-0.912) between SMI and CEUS in the grading of neovessels within the carotid plaques at the plaque level (n=104) (Figure 2). Of note, eight plaques were classified as grade 1 on SMI, but no bubbles were found on CEUS (Figure 3). In contrast, only one plaque was classified as grade 1 on CEUS, but no visible blood flow signal was observed on SMI. At the patient level $(\mathrm{n}=78)$, weighted kappa analysis showed excellent agreement $(\kappa=0.820,95 \%$ CI: $0.720-0.920$ ) between SMI and CEUS in the grading of neovessels within the carotid plaques.

\section{Agreement between SMI and histology}

Of the total 78 patients, $27(34.6 \%)$ underwent carotid endarterectomy, but 2 were excluded due to severely damaged specimens. Therefore, a total of 25 (32.1\%) histologic specimens of carotid plaques from 25 individual patients were obtained for analysis. The plaque characteristics of the 25 patients who underwent SMI and histologic examination are detailed in Table 4. Neovessels were observed in all 25 plaques on histology and the mean value of the neovessel density was $5.6 \pm 4.9$ neovessels $/ \mathrm{mm}^{2}$. There was a strong correlation $(\mathrm{r}=0.660, \mathrm{P}<0.001)$ between SMI and histology in the grading of neovessels within the carotid plaques. Figure 4 represents an example of good agreement between SMI and histology in visualizing neovessels within the carotid plaques. A strong correlation was also found between CEUS and histology $(r=0.618$, $\mathrm{P}=0.001)$ in the grading of neovessels within carotid plaques.

\section{Reproducibility of SMI measurement}

SMI was found to have excellent scan-rescan agreement in the evaluation of neovessels within carotid plaques $(\kappa=0.857$, 95\% CI: $0.588-1.000)$. The weighted kappa values for the intra-observer and inter-observer agreement of the 


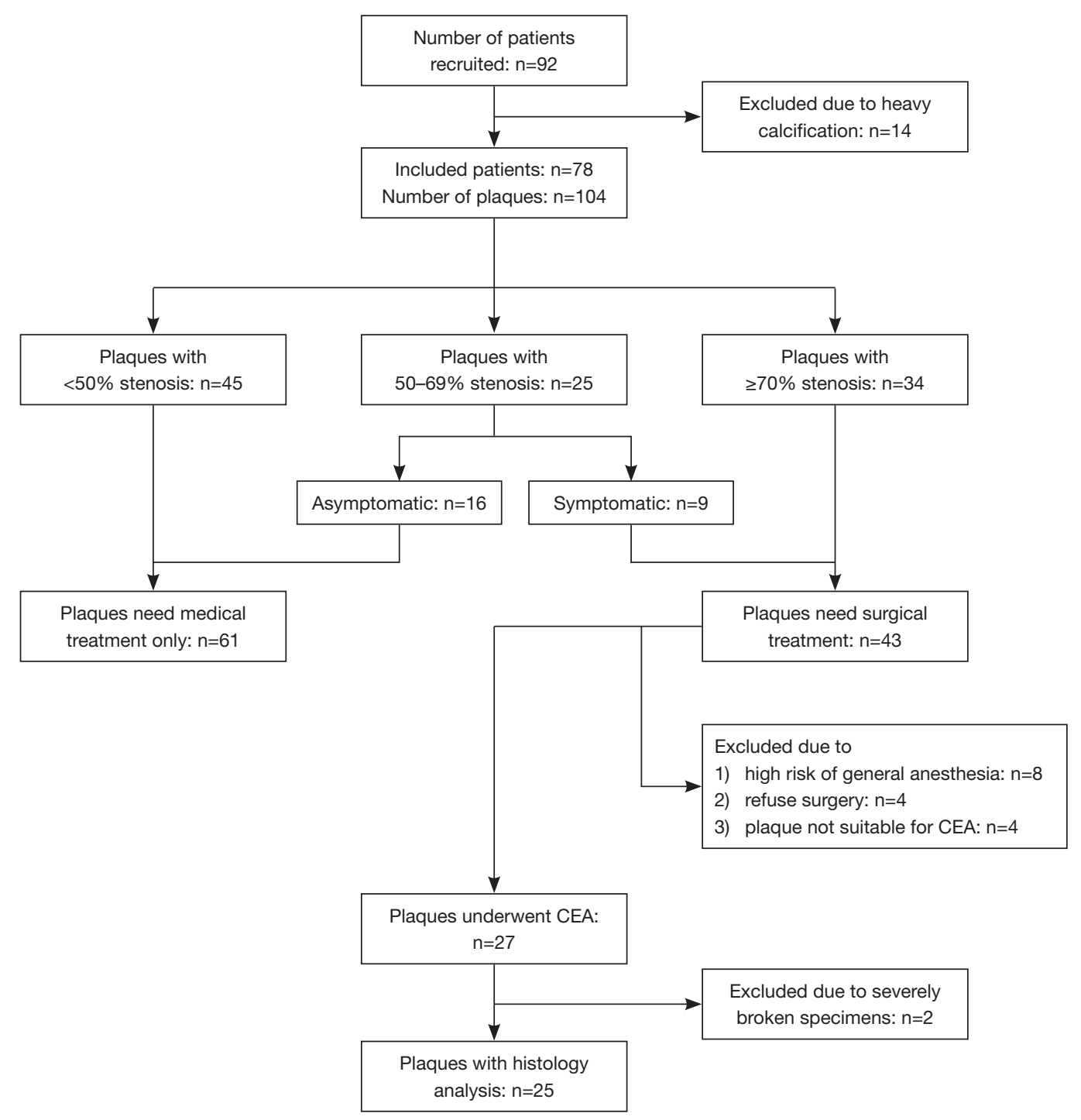

Figure 1 Flow diagram of patient recruitment process. CEA, carotid endarterectomy.

assessment of blood flow signals with SMI were 0.810 (95\% CI: $0.563-1.000)$ and 0.754 (95\% CI: 0.441-1.000), respectively.

\section{Discussion}

This study investigated the usefulness of SMI in assessing neovessels within carotid plaques validated by CEUS and histology. Excellent agreement was found between SMI and CEUS, and good agreement was found between SMI and histology in the grading of carotid intraplaque neovessels. We also found that SMI detected more intraplaque neovessels than CEUS. Our findings suggest that SMI could be an alternative non-contrast-enhanced imaging approach for the evaluation of neovessels within atherosclerotic plaques.

In the present study, SMI showed excellent agreement with CEUS in the assessment of intraplaque neovessels. In a recent study by Oura et al., investigators recruited 27 patients with carotid plaques (intima-media thickness $\geq 2 \mathrm{~mm})$ and found fair agreement $(\kappa=0.5)$ between SMI and CEUS in detecting neovessels (25). In another study by Zhu et al., 82 patients with carotid plaques were recruited, 
Table 1 Baseline clinical characteristics of 78 patients

\begin{tabular}{lcc}
\hline Characteristics & Mean \pm SD, or $n(\%)$ & Range \\
\hline Age, years & $67.3 \pm 8.9$ & $42-87$ \\
Sex, male & $63(80.8)$ & - \\
BMl, kg/m & $24.9 \pm 3.4$ & $17.9-37.0$ \\
Smoke & $39(50.0)$ & - \\
Hypertension & $59(75.6)$ & - \\
SBP, mmHg & $137.8 \pm 21.0$ & $93-202$ \\
DBP, mmHg & $72.3 \pm 12.3$ & $45-110$ \\
Hyperlipidemia & $46(59.0)$ & - \\
Statin use & $22(28.2)$ & - \\
LDL, mmol/L & $2.5 \pm 0.9$ & $1.0-4.5$ \\
HDL, mmol/L & $1.0 \pm 0.3$ & $0.5-1.9$ \\
TC, mmol/L & $4.2 \pm 1.0$ & $2.4-6.7$ \\
TG, mmol/L & $1.5 \pm 0.9$ & $0.6-6.1$ \\
Diabetes mellitus & $28(35.9)$ & - \\
CHD & $36(46.2)$ & - \\
Stroke & $23(29.5)$ & -
\end{tabular}

SD, standard deviation; BMI, body mass index; SBP, systolic blood pressure; DBP, diastolic blood pressure; LDL, low-density lipoprotein; HDL, high-density lipoprotein; TC, total cholesterol; $\mathrm{TG}$, triglycerides; $\mathrm{CHD}$, coronary heart disease.

and good consistency $(\kappa>0.75)$ between SMI and CEUS was observed (26). Our study with a larger sample size $(n=104)$ showed better agreement between SMI and CEUS than previous reports. The present study also showed that SMI was able to detect more neovessels, particularly small amounts of neovascularization, compared to CEUS. This may be due to the nature of SMI, which allows repeated scans to be carried out at multiple different sections and orientations. In contrast, during CEUS examination, only one section can be assigned to monitor the microbubbles in one bolus injection of contrast agent.

To the best of our knowledge, this is one of the first studies to validate the usefulness of SMI in evaluating intraplaque neovessels through comparison with histology. In a recent study by Chen et al., neovessels in carotid atherosclerotic plaques were assessed in 28 patients, and a significant correlation $(\mathrm{r}=0.788, \mathrm{P}<0.001)$ was found between SMI and histology (27). In addition to the agreement of
Table 2 Ultrasound characteristics of carotid plaques ( $\mathrm{n}=104)$

\begin{tabular}{lcc}
\hline Characteristics & Mean \pm SD, or n (\%) & Range \\
\hline Thickness, mm & $4.3 \pm 1.1$ & $2.6-8.5$ \\
Length, mm & $18.8 \pm 6.6$ & $6.0-37.8$ \\
Type & & \\
I & $40(38.5)$ & - \\
II & $43(41.3)$ & - \\
III & $13(12.5)$ & - \\
IV & $8(7.7)$ & - \\
Stenosis & & - \\
$<50 \%$ & $45(43.3)$ & - \\
$50-69 \%$ & $25(24.0)$ & - \\
$\geq 70 \%$ & $34(32.7)$ & - \\
SMI & & - \\
Grade 0 & $19(18.3)$ & - \\
Grade 1 & $53(51.0)$ & - \\
Grade 2 & $32(30.8)$ & - \\
\hline
\end{tabular}

SD, standard deviation; SMI, superb microvascular imaging.

Table 3 Agreement between SMI and CEUS in the assessment of neovessels $(\mathrm{n}=104)$

\begin{tabular}{lcccc}
\hline \multirow{2}{*}{ SMI } & \multicolumn{3}{c}{ CEUS } & Total \\
\cline { 2 - 4 } & Grade 0 & Grade 1 & Grade 2 & \\
\hline Grade 0 & 18 & 1 & 0 & 19 \\
Grade 1 & 8 & 42 & 3 & 53 \\
Grade 2 & 0 & 2 & 30 & 32 \\
Total & 26 & 45 & 33 & 104 \\
\hline
\end{tabular}

Weighted $\kappa=0.825,95 \% \mathrm{Cl}: 0.739-0.912$. SMI, superb microvascular imaging; CEUS, contrast-enhanced ultrasound.

SMI and histology, we also investigated the consistency between SMI and CEUS using a larger sample size. In the present study, good agreement was found between SMI and histology in the grading of carotid intraplaque neovessels. Technically, only microvessels with a blood flow velocity $\geq 0.4 \mathrm{~cm} / \mathrm{s}$ can be captured by SMI. This may explain the phenomenon in some cases in which neovessels were detected by histology but cannot be delineated by SMI. 

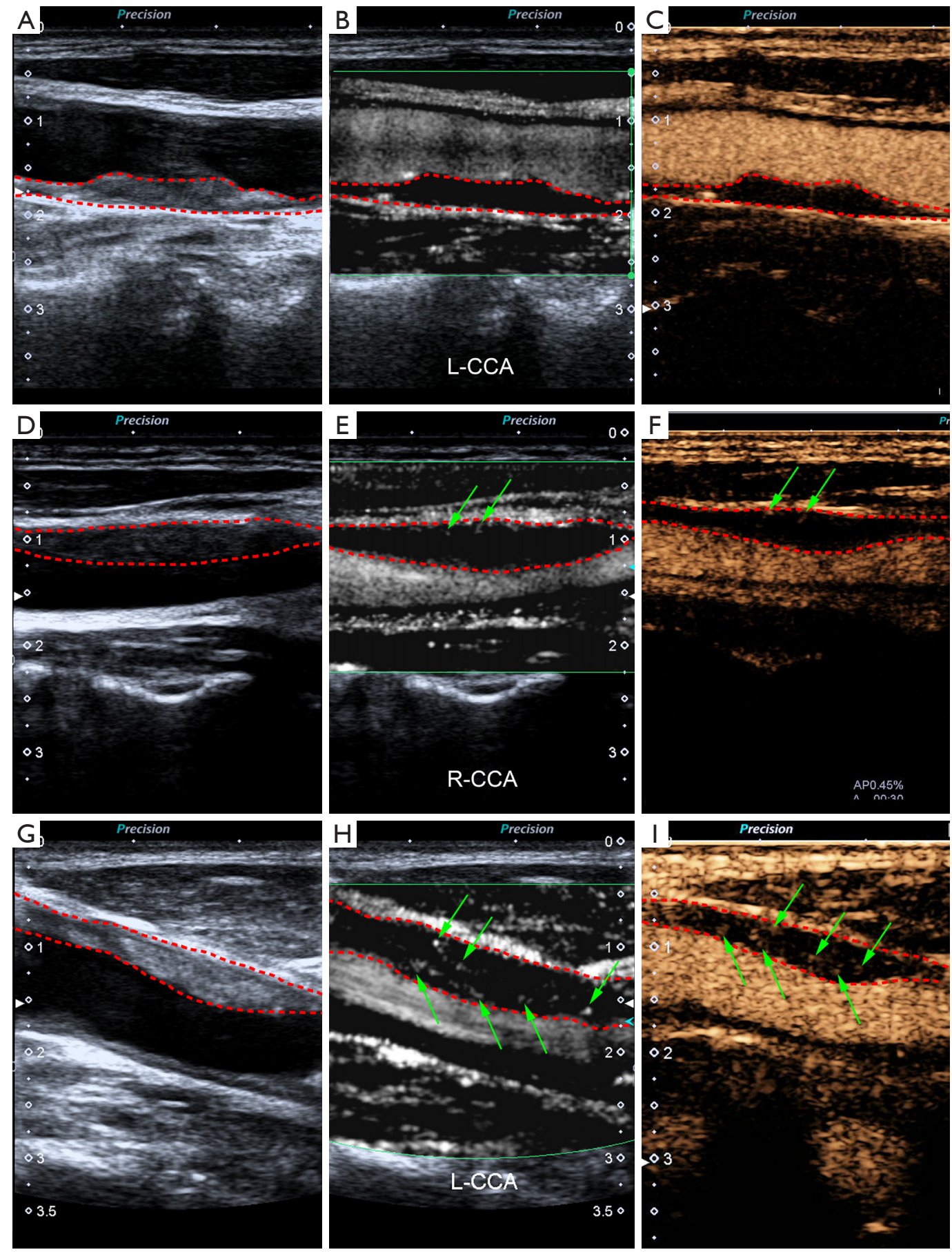

Figure 2 Patient 1: a 78-year-old man with left carotid atherosclerotic plaque (A-C). The B-mode image shows an atherosclerotic plaque with predominant hypoecho (A). No neovessel was detected in the carotid plaque of patient 1 neither by SMI (B) nor by CEUS imaging (C). The neovessel within the plaque was classified as grade 0. Patient 2: a 54-year-old man with right carotid atherosclerotic plaque (D-F). The grayscale ultrasound shows a uniform anechoic atherosclerotic plaque (D). Neovessels within the plaques are indicated by green arrows and classified as grade 1 on both SMI (E) and CEUS imaging (F). Patient 3: an 80-year-old man with left carotid atherosclerotic plaque (G-I). The B-mode image shows an atherosclerotic plaque with uniform hyperecho $(\mathrm{G})$. Neovessels within the plaques are indicated by green arrows and classified as grade 2 on both SMI (H) and CEUS imaging (I). SMI, superb microvascular imaging; CEUS, contrast-enhanced ultrasound. 

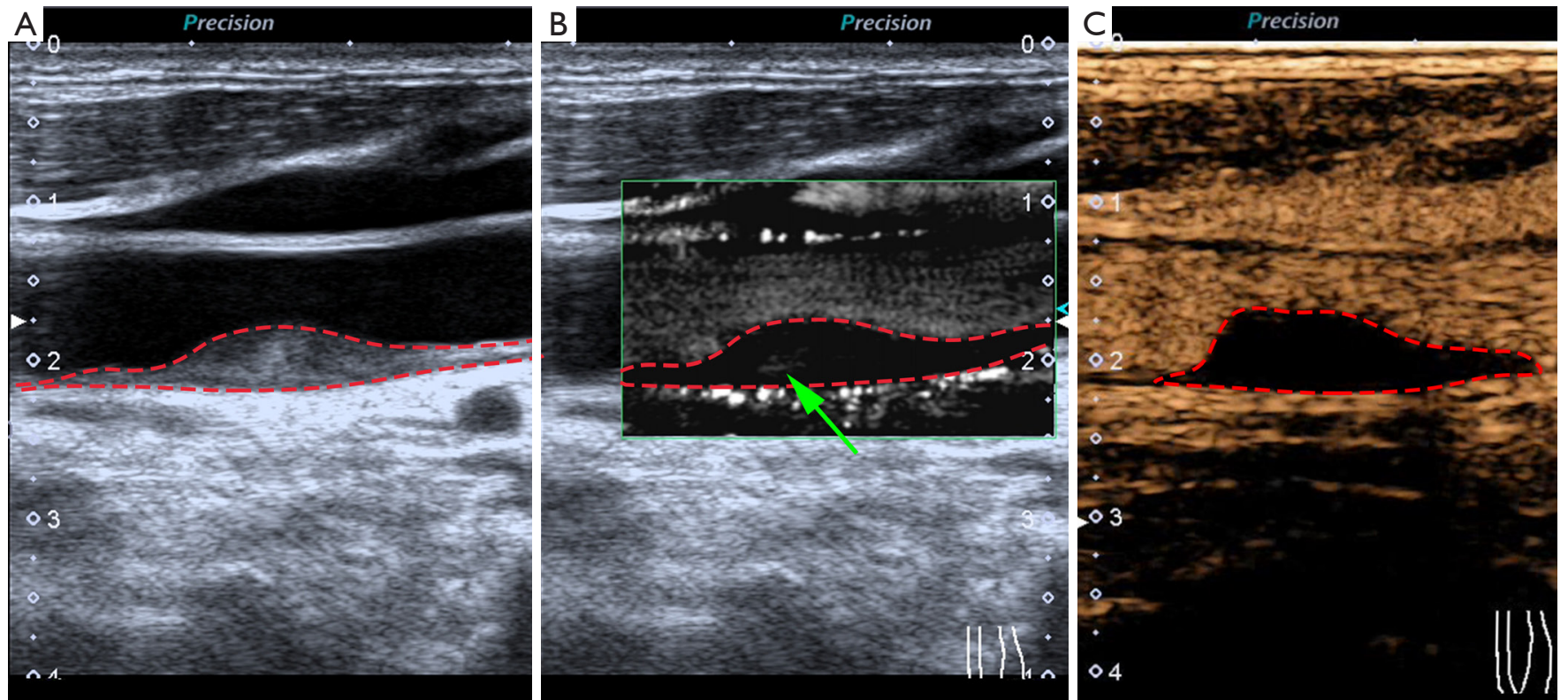

Figure 3 Scans from a 70-year-old man with left carotid atherosclerotic plaque. Grayscale ultrasound shows the longitudinal section of the plaque (A). The neovessels were detectable by SMI (B, grade 1) but invisible on CEUS (C, grade 0). Neovessels within the plaques are indicated by green arrows (B). SMI, superb microvascular imaging; CEUS, contrast-enhanced ultrasound.

In the present study, the histologic sections were taken from plaque specimens every $2 \mathrm{~mm}$, which may lead to an underestimation of intraplaque neovessels.

We found that SMI had excellent scan-rescan, interreader and intra-reader reproducibility in evaluating neovessels within carotid plaques. In the present study, the neovessels in carotid plaques were classified into three grades, which differs from a previous study in which only the presence or absence of neovessels was determined (25). Since SMI does not require the administration of a contrast agent, repeated scans at multiple orientations can be performed and comprehensive evaluation can be achieved. This may ensure an excellent reproducibility for operators. The excellent scan-rescan agreement of SMI suggests that this is a highly reproducible technique which can be applied to monitor the natural history or treatment effects of carotid plaques in future studies.

Without the administration of a contrast agent, SMI could be a simple and effective imaging modality for the clinical screening and follow-up of unstable carotid plaques. Through a rapid SMI scan of less than 1 minute, radiologists could be able to provide more information about the vulnerability of carotid plaques to physicians. In future research, we intend to explore whether carotid plaque neovascularization assessed with SMI can help to predict future cerebrovascular events. Furthermore, SMI could also be utilized to monitor the effects of treatment on carotid plaques.

Our study has several limitations. First, due to the difficulties in performing CEUS for plaques with a thinner wall, only carotid plaques with a maximum plaque thickness $>2.5 \mathrm{~mm}$ were included in this study. Therefore, this study lacks early plaques without neovessels as a control. Second, it is challenging to accurately match the sections of SMI with histology specimens because of the different orientations of longitudinal view with SMI and the crosssectional view with histology. Future studies using SMI with a cross-sectional view, which will better conform to histologic sections, are warranted. Third, neovessels with a blood flow velocity $<0.4 \mathrm{~cm} / \mathrm{s}$ cannot be captured by the current SMI technique. It is hoped that this resolution can be improved in the future by utilizing novel techniques. Fourth, the evaluations of SMI and CEUS imaging in our study were semi-quantitative. Time-intensity curves on CEUS imaging were able to provide quantitative parameters for assessing neovascularization within the carotid plaques $(10,32)$; however, the quantitative technology for SMI was 
Table 4 SMI, CEUS, and histologic characteristics of 25 patients with carotid plaques

\begin{tabular}{|c|c|c|c|c|c|c|c|c|}
\hline $\begin{array}{l}\text { Patient } \\
\text { no. }\end{array}$ & Sex/age (years) & Side & \multicolumn{4}{|c|}{ Ultrasound } & \multicolumn{2}{|c|}{ Histology } \\
\hline 2 & $\mathrm{M} / 63$ & $\mathrm{R}$ & 19.6 & 4.8 & 2 & 2 & 16.9 & 22.7 \\
\hline 5 & $F / 66$ & $\mathrm{R}$ & 19.1 & 4.3 & 1 & 1 & 16.1 & 4.3 \\
\hline 6 & $\mathrm{M} / 60$ & $\mathrm{R}$ & 17.1 & 4.9 & 0 & 1 & 14.9 & 0.3 \\
\hline 7 & M/65 & $\mathrm{R}$ & 13.1 & 5.7 & 1 & 1 & 11.6 & 2.0 \\
\hline 11 & M/63 & $\mathrm{L}$ & 14.1 & 8.5 & 1 & 2 & 14.7 & 8.4 \\
\hline 12 & $\mathrm{M} / 62$ & $\mathrm{~L}$ & 20.5 & 5.6 & 1 & 1 & 20.2 & 0.4 \\
\hline 13 & $F / 69$ & $\mathrm{R}$ & 21.7 & 4.7 & 2 & 2 & 19.9 & 6.4 \\
\hline 14 & $M / 71$ & $\mathrm{R}$ & 24.6 & 5.1 & 2 & 2 & 21.5 & 7.6 \\
\hline 15 & $M / 71$ & $\mathrm{~L}$ & 22.0 & 2.9 & 2 & 2 & 20.5 & 4.5 \\
\hline 16 & $\mathrm{M} / 72$ & $\mathrm{R}$ & 23.5 & 6.1 & 1 & 1 & 20.9 & 3.4 \\
\hline 17 & $F / 42$ & $\mathrm{~L}$ & 29.0 & 5.1 & 2 & 2 & 26.9 & 14.3 \\
\hline 23 & $\mathrm{M} / 73$ & $\mathrm{R}$ & 16.2 & 2.6 & 2 & 2 & 14.4 & 5.6 \\
\hline 24 & $\mathrm{M} / 66$ & $\mathrm{~L}$ & 28.6 & 3.6 & 1 & 1 & 25.3 & 1.3 \\
\hline 25 & $\mathrm{~F} / 72$ & $\mathrm{~L}$ & 29.1 & 4.1 & 1 & 1 & 26.7 & 2.9 \\
\hline
\end{tabular}

Neovessel density was measured by histology. R: right carotid artery; L: left carotid artery. SMI, superb microvascular imaging; CEUS, contrast-enhanced ultrasound.

not available in the present study. This might be a future research direction. Fifth, the histology sample size was small. Finally, because of the shadow caused by calcification, type $\mathrm{V}$ plaques on ultrasound imaging were excluded in this study. Therefore, the results of this study cannot be translated to all patients with carotid stenosis. DCE-MRI may be an appropriate tool for the evaluation of neovessels in type $\mathrm{V}$ plaques.

\section{Conclusions}

SMI is capable of reliably characterizing neovessels within 

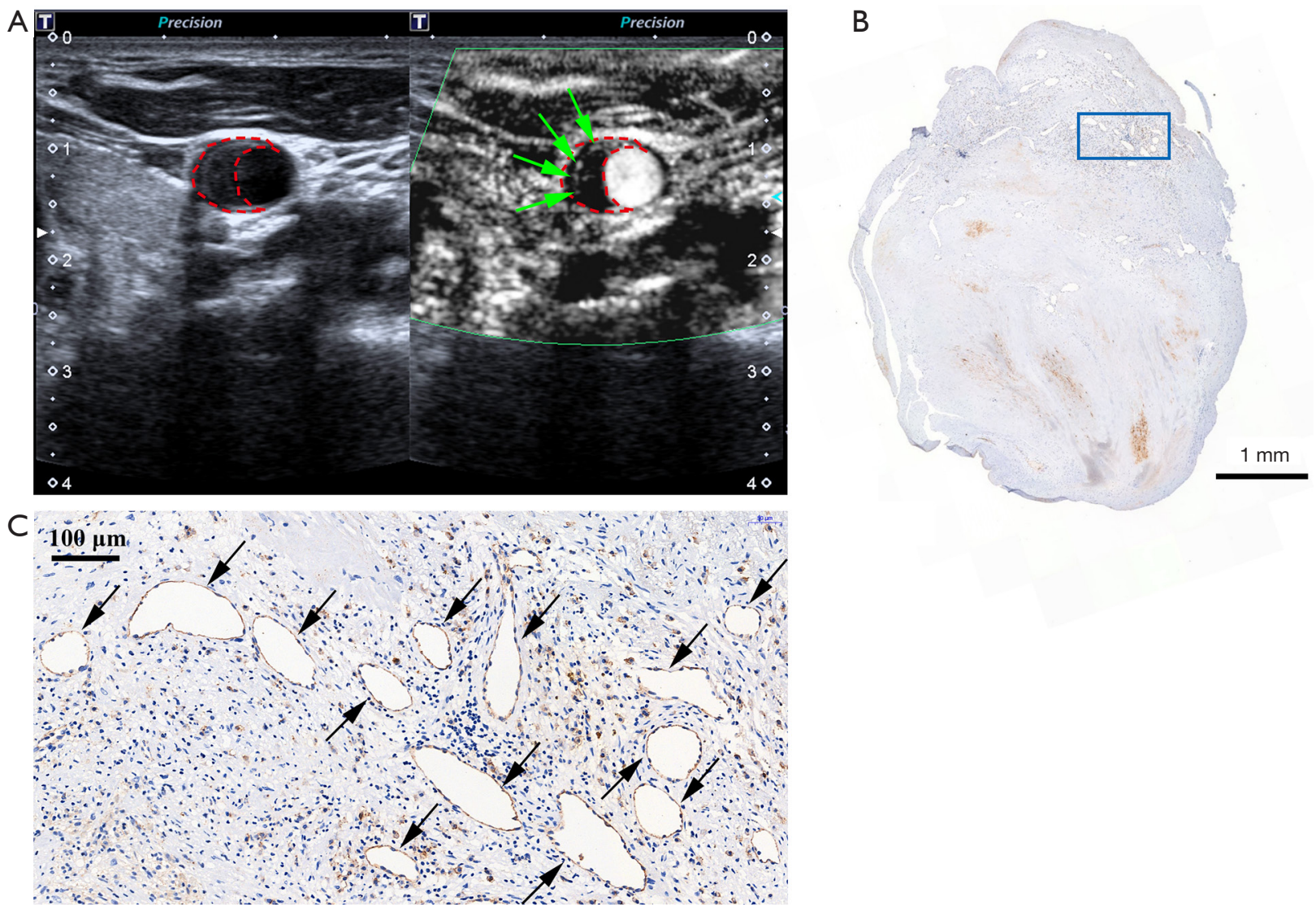

Figure 4 SMI images and histologic section from the same 76-year-old male patient. (A) A transverse section of carotid plaque: left: grayscale ultrasound; right: SMI image, extensive intraplaque blood flow signals. Neovessels within the plaques are indicated by green arrows. (B) Histologic section from the CEA specimen with CD31 stain (magnification, $\times 40$ ), and marked neovessels can be seen. (C) Highpower view of the histologic section from the same specimen with CD31 stain (magnification, $\times 480$ ). Neovessels within the plaques are indicated by black arrows. SMI, superb microvascular imaging; CEA, carotid endarterectomy

carotid atherosclerotic plaques and has good and excellent agreement with histology and CEUS, respectively.

\section{Acknowledgments}

Funding: This study is supported by grants from the Beijing Tsinghua Changgung Hospital Fund (No. 12016C1005, 12015C1029), the National Natural Science Foundation of China (81771825), the Beijing Municipal Science and Technology Commission (D17110003017003), and the Ministry of Science and Technology of China (2017YFC1307904).

\section{Footnote}

Conflicts of Interest: All authors have completed the ICMJE uniform disclosure form (available at http://dx.doi. org/10.21037/qims-20-933). The authors have no conflicts of interest to declare.

Ethical Statement: The study was approved by the institutional ethics committee of Beijing Tsinghua Changgung Hospital and informed consent was obtained from all patients.

Open Access Statement: This is an Open Access article distributed in accordance with the Creative Commons Attribution-NonCommercial-NoDerivs 4.0 International License (CC BY-NC-ND 4.0), which permits the noncommercial replication and distribution of the article with the strict proviso that no changes or edits are made and the original work is properly cited (including links to both the 
formal publication through the relevant DOI and the license). See: https://creativecommons.org/licenses/by-nc-nd/4.0/.

\section{References}

1. Chaturvedi S, Bruno A, Feasby T, Holloway R, Benavente O, Cohen S N, Cote R, Hess D, Saver J, Spence J D, Stern B, Wilterdink J. Carotid endarterectomy--an evidencebased review: report of the Therapeutics and Technology Assessment Subcommittee of the American Academy of Neurology. Neurology 2005;65:794-801.

2. Moreno PR, Purushothaman KR, Sirol M, Levy AP, Fuster V. Neovascularization in human atherosclerosis. Circulation 2006;113:2245-52.

3. Sharma P, Kochar P, Soin P, Cohen S. Bisystolic vertebral artery: critical finding or can be ignored? J Clin Imaging Sci 2019;9:2.

4. Sharma P, Hegde R, Kulkarni A, Sharma S, Soin P, Kochar PS, Kumar Y. Traumatic vertebral artery injury: a review of the screening criteria, imaging spectrum, mimics, and pitfalls. Pol J Radiol 2019;84:e307-18.

5. Qiao H, Cai Y, Huang M, Liu Y, Zhang Q, Huang L, Chen H, Yuan C, Zhao X. Quantitative assessment of carotid artery atherosclerosis by three-dimensional magnetic resonance and two-dimensional ultrasound imaging: a comparison study. Quant Imaging Med Surg 2020;10:1021-32.

6. Kerwin W, Hooker A, Spilker M, Vicini P, Ferguson M, Hatsukami T, Yuan C. Quantitative magnetic resonance imaging analysis of neovasculature volume in carotid atherosclerotic plaque. Circulation 2003;107:851-6.

7. Kerwin WS, O'Brien KD, Ferguson MS, Polissar N, Hatsukami TS, Yuan C. Inflammation in carotid atherosclerotic plaque: a dynamic contrast-enhanced MR imaging study. Radiology 2006;241:459-68.

8. Kerwin WS, Oikawa M, Yuan C, Jarvik GP, Hatsukami TS. MR imaging of adventitial vasa vasorum in carotid atherosclerosis. Magn Reson Med 2008;59:507-14.

9. Hoogi A, Adam D, Hoffman A, Kerner H, Reisner S, Gaitini D. Carotid plaque vulnerability: Quantification of neovascularization on contrast-enhanced ultrasound with histopathologic correlation. AJR Am J Roentgenol 2011;196:431-6.

10. Li C, He W, Guo D, Chen L, Jin X, Wang W, Huang B, Wang W. Quantification of carotid plaque neovascularization using contrast-enhanced ultrasound with histopathologic validation. Ultrasound Med Biol 2014;40:1827-33.
11. Coli S, Magnoni M, Sangiorgi G, Marrocco-Trischitta MM, Melisurgo G, Mauriello A, Spagnoli L, Chiesa R, Cianflone D, Maseri A. Contrast-enhanced ultrasound imaging of intraplaque neovascularization in carotid arteries: correlation with histology and plaque echogenicity. J Am Coll Cardiol 2008;52:223-30.

12. Xu B, Xing J, Wu W, Zhang WJ, Zhu QQ, Zhang D, Sun NN, Wu C, Kang GJ, Zhai L, Li WD, Meng Y, Du TY. Improved plaque neovascularization following 2-year atorvastatin therapy based on contrast-enhanced ultrasonography: A pilot study. Exp Ther Med 2018;15:4491-7.

13. Dong L, Kerwin WS, Chen H, Chu B, Underhill HR, Neradilek MB, Hatsukami TS, Yuan C, Zhao XQ. Carotid artery atherosclerosis: effect of intensive lipid therapy on the vasa vasorum--evaluation by using dynamic contrastenhanced MR imaging. Radiology 2011;260:224-31.

14. Superb Micro-Vascular Imaging (SMI). Toshiba Medical Systems. Available online: https://us.medical.canon/ products/ultrasound/aplio-i-series/technology (Accessed 5 October 2020).

15. Machado P, Segal S, Lyshchik A, Forsberg F. A novel microvascular flow technique: initial results in thyroids. Ultrasound Q 2016;32:67-74.

16. Kong J, Li JC, Wang HY, Wang YH, Zhao RN, Zhang $\mathrm{Y}$, Jin J. Role of superb micro-vascular imaging in the preoperative evaluation of thyroid nodules: Comparison with power doppler flow imaging. J Ultrasound Med 2017;36:1329-37.

17. Lu R, Meng Y, Zhang Y, Zhao W, Wang X, Jin M, Guo R. Superb microvascular imaging (SMI) compared with conventional ultrasound for evaluating thyroid nodules. BMC Med Imaging 2017;17:65.

18. Bakdik S, Arslan S, Oncu F, Durmaz MS, Altunkeser A, Eryilmaz MA, Unlu Y. Effectiveness of Superb Microvascular Imaging for the differentiation of intraductal breast lesions. Med Ultrason 2018;20:306-12 .

19. Zhu YC, Zhang Y, Deng SH, Jiang Q. Diagnostic performance of superb microvascular imaging (SMI) combined with shear-wave elastography in evaluating breast lesions. Med Sci Monit 2018;24:5935-42.

20. Yongfeng Z, Ping Z, Wengang L, Yang S, Shuangming T. Application of a novel microvascular imaging technique in breast lesion evaluation. Ultrasound Med Biol 2016;42:2097-105.

21. Park AY, Seo BK, Cha SH, Yeom SK, Lee SW, Chung $\mathrm{HH}$. An innovative ultrasound technique for evaluation of tumor vascularity in breast cancers: superb Micro-Vascular 
imaging. J Breast Cancer 2016;19:210-3.

22. Dubinsky TJ, Revels J, Wang S, Toia G, Sonneborn R, Hippe DS, Erpelding T. Comparison of superb microvascular imaging with color flow and power doppler imaging of small hepatocellular carcinomas. J Ultrasound Med 2018;37:2915-24.

23. Goeral K, Hojreh A, Kasprian G, Klebermass-Schrehof K, Weber M, Mitter C, Berger A, Prayer D, Brugger PC, Vergesslich-Rothschild K, Patsch JM. Microvessel ultrasound of neonatal brain parenchyma: feasibility, reproducibility, and normal imaging features by superb microvascular imaging (SMI). Eur Radiol 2019;29:2127-36.

24. Yang DB, Zhou J, Feng L, Xu R, Wang YC. Value of superb micro-vascular imaging in predicting ischemic stroke in patients with carotid atherosclerotic plaques. World J Clin Cases 2019;7:839-48.

25. Oura K, Kato T, Ohba H, Terayama Y. Evaluation of intraplaque neovascularization using superb microvascular imaging and contrast-enhanced ultrasonography. J Stroke Cerebrovasc Dis 2018;27:2348-53.

26. Zhu YC, Jiang XZ, Bai QK, Deng SH, Zhang Y, Zhang $\mathrm{ZP}$, Jiang Q. Evaluating the efficacy of atorvastatin on patients with carotid plaque by an innovative ultrasonography. J Stroke Cerebrovasc Dis 2019;28:830-7.

27. Chen X, Wang H, Jiang Y, Li J, Li N, Kong J, Zhang $\mathrm{X}$, Ye W, Zhao D, Cai S. Neovascularization in carotid atherosclerotic plaques can be effectively evaluated by superb microvascular imaging (SMI): Initial experience.

Cite this article as: Meng Q, Xie X, Li L, Jiang C, Zhao K, Bai Z, Zheng Z, Yang Y, Yu Y, Zhang H, Zhao X. Assessment of neovascularization of carotid artery atherosclerotic plaques using superb microvascular imaging: a comparison with contrast-enhanced ultrasound imaging and histology. Quant Imaging Med Surg 2021;11(5):1958-1969. doi: 10.21037/qims20-933
Vasc Med 2020;25:328-33.

28. Grant EG, Benson CB, Moneta GL, Alexandrov AV, Baker JD, Bluth EI, Carroll BA, Eliasziw M, Gocke J, Hertzberg BS, Katanick S, Needleman L, Pellerito J, Polak JF, Rholl KS, Wooster DL, Zierler RE. Carotid artery stenosis: gray-scale and Doppler US diagnosis--Society of Radiologists in Ultrasound Consensus Conference. Radiology 2003;229:340-6.

29. Geroulakos G, Ramaswami G, Nicolaides A, James K, Labropoulos N, Belcaro G, Holloway M. Characterization of symptomatic and asymptomatic carotid plaques using high-resolution real-time ultrasonography. Br J Surg 1993;80:1274-7.

30. de Bray JM, Baud JM, Delanoy P, Camuzat JP, Dehans V, Descamp-Le Chevoir J, Launay JR, Luizy F, Sentou Y, Cales P. Reproducibility in ultrasonic characterization of carotid plaques. Cerebrovasc Dis 1998;8:273-7.

31. Deyama J, Nakamura T, Takishima I, Fujioka D, Kawabata K, Obata JE, Watanabe K, Watanabe Y, Saito Y, Mishina $\mathrm{H}$, Kugiyama K. Contrast-enhanced ultrasound imaging of carotid plaque neovascularization is useful for identifying high-risk patients with coronary artery disease. Circ J 2013;77:1499-507.

32. Saito K, Nagatsuka K, Ishibashi-Ueda H, Watanabe A, Kannki H, Iihara K. Contrast-enhanced ultrasound for the evaluation of neovascularization in atherosclerotic carotid artery plaques. Stroke 2014;45:3073-5. 